\title{
Bubble Motion under Gravity through Lattice Boltzmann Method
}

\author{
D.M. Nie \\ Institute of Fluid Mechanics \\ China Jiliang University \\ Hangzhou, 310018, China
}

\author{
L.M. Qiu \\ Institute of Refrigeration and Cryogenics \\ Zhejiang University \\ Hangzhou, 310027, China
}

\author{
X.B. Zhang \\ Institute of Refrigeration and Cryogenics \\ Zhejiang University \\ Hangzhou, 310027, China
}

\begin{abstract}
The motion of two bubbles under gravity is numerically studied through the lattice Boltzmann method for the Eotvos number ranging from 1 to 12. The effects of Eotvos number on the bubble coalescence and rising velocity are investigated. It has been found that the uppermost bubble deforms the most because of the maximum drag.In addition, the averaged rising velocity of the bubbles is also studied in this work.
\end{abstract}

\section{Keywords-lattice Boltzmann method; bubble; coalescence}

\section{INTRODUCTION}

Bubble motion is one ofthe most common gas-liquid flow phenomena and plays an important role in many industrial applications, such as cavitation fluid machinery,nucleate boiling in reactors and condenser/evaporator[1]. The motion of bubble under gravity is complex due to bubble deformation, coalescence and breakup.Understanding thedynamic interaction between bubbles is an important aspect ofthe design and operation of many industrial applications.

A number of investigations of the bubble motion in liquid have been conducted in the past [2-5] due to its scientific and engineering importance. In recent decades, the lattice Boltzmann method (LBM) has proved to be a powerful numerical scheme for the simulation of multiphase flow which is based on mesoscopicparticle dynamics. Its kinetic nature can provide many of the advantagesof molecular dynamics [6]. Several kinds of lattice Boltzmann model for simulating multiphase fluid have been established and applied to the simulations of gas bubbles under gravitysuccessfully. These models include potential method proposed by Shan et al. [7], color method proposed by Rothman et al. [8], and free energy method proposed by Swift et al. [9] and improved byZheng et al. [10]. The free energy method [10] was proved to be a good tool for the study of two-phase flows with high viscosity ratios and high density ratio.As shown by Gupta et al. [11] and $\mathrm{Yu}$ et al. [12], in comparison with the case of single bubble, the motion of multiple bubbles under gravity could be much more complex due to hydrodynamic interactions between bubbles. Besides, the bubble coalescence and break-up could take place occasionally which have great effect on the motion of multiple bubbles. However,a detailed numerical study of the influence of bubble collision and coalescence on the rising velocity has not been undertaken. It is important to focus on the fundamental understanding of the bubble collision and coalescence when multiple bubbles are rising under gravity.To fulfil this task the LBM proposed by Zheng et al. [10] is adopted in this work to study the rise behavior of multiple bubbles which are initially placed in in-line arrangement.This study will evaluate the coalescence patternand rising velocity of the bubbleswhich not only depend on the computational parameters but also depend on the initial arrangements. In addition, the terminal velocity is compared with the result of single bubble under the same conditions to illustrate the influence of multiple bubbles motion.

\section{NUMERICAL METHOD}

The discrete lattice Boltzmann equationsunder external forces for the continuity and momentum equations are given by,

$$
f_{i}\left(\boldsymbol{x}+\boldsymbol{e}_{i} \Delta t, t+\Delta t\right)-f_{i}(\boldsymbol{x}, t)=-\frac{1}{\tau_{n}}\left[f_{i}(\boldsymbol{x}, t)-f_{i}^{(\mathrm{eqq})}(\boldsymbol{x}, t)\right]+\frac{w_{i}}{c_{s}^{2}}\left(\boldsymbol{F}+\mu_{\phi} \nabla \phi\right) \cdot \boldsymbol{e}_{i}
$$

where $\mathrm{fi}(\mathrm{x}, \mathrm{t})$ is the density distribution function at the ith microscopic velocity ei,fi(eq)(x, t) is the equilibrium distribution function,Fis the external force(which is gravity in this work), $\Delta \mathrm{t}$ is the time step, $\tau$ nisthe relaxation time, cs is the speed of sound and wi aretheweights related to the D2Q9 model. is the order parameter that is responsible for the gas-liquid interface. is the chemical potential, which is defined in the following. The macroscopic variables $n$ and $u$ are determined by the distribution function as follows,

$$
n=\sum_{i} f_{i}, \quad n \boldsymbol{u}=\sum_{i} f_{i} \boldsymbol{e}_{i}
$$

The variables $\mathrm{n}$ and $\quad$ are defined as

$$
n=\frac{\rho_{L}+\rho_{G}}{2}, \quad \phi=\frac{\rho_{L}-\rho_{G}}{2}
$$


where L and $\mathrm{G}$ are the densities of liquid and gas phase, respectively. The equilibrium distribution functions fi (eq) are defined as

$$
f_{i}^{e q}=w_{i} A_{i}+w_{i} n\left[3 \boldsymbol{u} \cdot \boldsymbol{e}_{i}-\frac{3}{2} u^{2}+\frac{9}{4}\left(\boldsymbol{u} \cdot \boldsymbol{e}_{i}\right)^{2}\right]
$$

According to Zheng et al. [12], the coefficients are defined as

$$
A_{0}=\frac{9}{4} n-\frac{15}{4}\left(\phi \mu_{\phi}+\frac{1}{3} n\right), \quad A_{1-8}=3\left(\phi \mu_{\phi}+\frac{1}{3} n\right)
$$

The discrete lattice Boltzmann equations for the interface capture equation are given by [12],

$$
g_{i}\left(\boldsymbol{x}+\boldsymbol{e}_{i} \Delta t, t+\Delta t\right)=q g_{i}(\boldsymbol{x}, t)+(1-q) g_{i}\left(\boldsymbol{x}+\boldsymbol{e}_{i} \Delta t, t\right)-\frac{1}{\tau_{\phi}}\left[g_{i}(\boldsymbol{x}, t)-g_{i}^{(\mathrm{ec})}(\boldsymbol{x}, t)\right]
$$

whereqis a constant coefficient, which is determined by,

$$
q=\frac{1}{\tau_{\phi}+0.5}
$$

The macroscopic variable, i.e. the order parameter , is given by,

$$
\phi=\sum_{i} g_{i}
$$

According to Zheng et al. [12], the chemical potential is computed using,

$$
\mu_{\phi}=A\left(4 \phi^{3}-4 \phi^{* 2} \phi\right)-\kappa \nabla^{2} \phi
$$

where $*=0.5(\mathrm{~L}+\mathrm{G})$, and $\mathrm{L}$ and $\mathrm{G}$ are the densities of the liquid phase and the gas phase, respectively. A and are parameters related to the thickness of the interface layer $\mathrm{W}$ and the surface tension coefficient, which are expressed as,

$$
W=\frac{\sqrt{2 \kappa / A}}{\phi^{*}}, \quad \sigma=\frac{4 \sqrt{2 \kappa A}}{3} \phi^{* 3}
$$

The equilibrium distribution functions gi(eq) are[12],

$$
g_{i}^{(\mathrm{eq})}=A_{i}+B_{i} \phi+C_{i} \phi \boldsymbol{e}_{i} \cdot \boldsymbol{u}
$$

where thecoefficientsAi, Bi and Ciare defined according to the D2Q5 lattice.

By Chapman-Enskoganalysis, the Navier-Stokes equations and an interface evolution equation can be obtained byEq. (1) and Eq.(6),

$$
\begin{gathered}
\frac{\partial n}{\partial t}+\nabla \cdot(n \boldsymbol{u})=0 \\
\frac{\partial(n \boldsymbol{u})}{\partial t}+\nabla \cdot(n \boldsymbol{u} \boldsymbol{u})=-\nabla P+\mu \nabla^{2} \boldsymbol{u}+\boldsymbol{F} \\
\frac{\partial \phi}{\partial t}+\nabla \cdot(\phi \mathbf{u})=\theta_{M} \nabla^{2} \mu_{\phi}
\end{gathered}
$$

where $\mathrm{M}$ is the mobility, given by $=\mathrm{q}(-0.5) \mathrm{x}$. The viscosity is $=(\mathrm{n}-0.5) \mathrm{n} / 3$.

\section{RESUlTS AND DISCUSSION}

Threecasesare carried out to validate the present computational code. The first is the Laplace law, which is given (for the two-dimensional case)by

$$
\Delta p=\frac{\sigma}{R}
$$

where $p$ is the pressure jump across the interface, and $R$ is the bubble radius. In this work, the Laplace law is validated by calculating the pressure jump while varying the bubble radius from $\mathrm{R}=10$ to $\mathrm{R}=80$. Other parameters are fixed at $\mathrm{L}=1000, \mathrm{G}=1, \quad=2$ and $=400$. The interface layerthickness is set to be $\mathrm{W}=4$ for $\mathrm{R}<20$ and $\mathrm{W}=5$ for $\mathrm{R} \geq 20$. The relaxation times are $\mathrm{n}=0.875$ and $=0.7$. The results are shown in Figure I, which showsgood agreement between the numerical results and the analytical solution of Eq.(15).

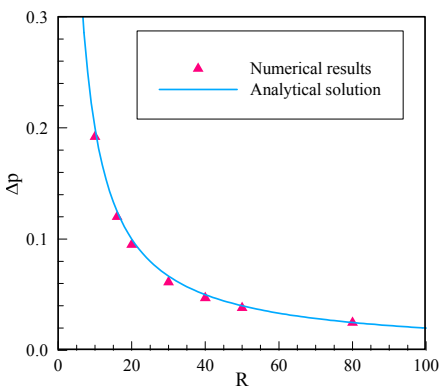

FIGURE I . THE VERIFICATION OF THE LAPLACE LAW.

In this sectionmultiple bubbles motion for both horizontal arrangement and vertical arrangement are investigated in detail. The parameters are fixed at $\mathrm{L}=2.6, \mathrm{G}=1, \quad=0.01$, $\mathrm{W}=4.5, \mathrm{R}=20$ and $=0.5$. The computational domain is $250 \times 1500$ and stationary walls are applied for all boundaries.

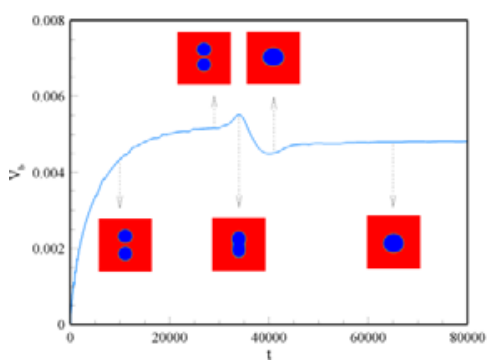

FIGURE II . TIME EVOLUTION OF THE RISING VELOCITY OF TWO BUBBLES FOR EO $=1$.

Figure II shows the time evolution of the rising velocity of two bubbles for $E 0=1$. The two bubbles are initially placed in a vertical orientationseparated by center to center distance of $2.5 \mathrm{R}$ initially. As shown in the figure, after an initial period of evolution the velocity becomes constant which indicates that the merged bubble is rising at a constant velocity due to the balance between buoyancy and drag forces. For better understanding the contours of order parameter which visualize the process of bubble collision and coalescence are also 
shown in Figure II. It's observed that an oscillation occurs in the bubble velocity before the steady state. Obviously, this oscillation corresponds to the bubble coalescence process. Furthermore, as shown in fig. 3 the terminal velocity is smaller than the velocity before the two bubbles collide and coalesce.

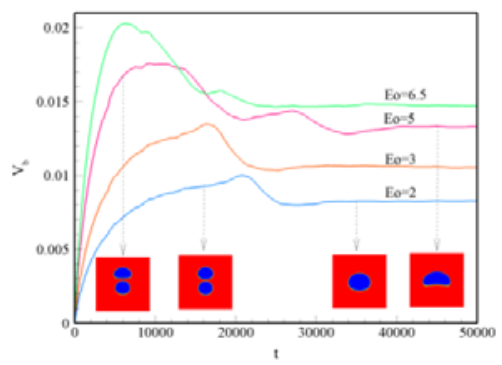

FIGURE III. TIME EVOLUTION OF THE RISING VELOCITY OF TWO BUBBLES FOR DIFFERENT EO.

The effects of the Eotvos number on the evolution of bubble velocity are shown in FigureIII. It's observed that as the Eotvos number increases the bubble velocity increases and the magnitude of oscillation also increases.Moreover, it has been found that in all cases $(1 \leq \mathrm{E} 0 \leq 12)$ the two bubbles would collide andcoalesce. The lower bubble always rises at a higher speed than the upper one because it is located at the wake behind the upper bubble. This leads to a smaller drag force for the lower bubble. Since the relative velocity of the bubbles is non-zero, the distance between the two bubbles keeps decreasing with time. Eventually the bubbles come to contact and merge, and soon after form a larger bubble with twice the volume as the initial bubble.

In order to present more insight into the rising process of multiple bubbles under gravity, the terminal velocities of two bubbles for different Eotvos numbersare shown in FigureIV. For the purpose of comparison, the results of a single bubble under the same conditions are also shown in the figure. It's unexpected that for very low Eotvos numbers such as Eo $\leq 3$, the terminal velocities of two bubbles are a little larger than those of a single bubble, as can be seen in Figure 4. The reason is not clear.However, forhigh Eotvos numberssuch as Eo $>6$ the terminal velocitiesare similar for all cases, indicating that the influence of the wall boundaries on the terminal velocity is insignificant. Moreover, the bubble coalescence strongly affects the time evolution of bubble velocity, but has little influence on the terminal velocity.

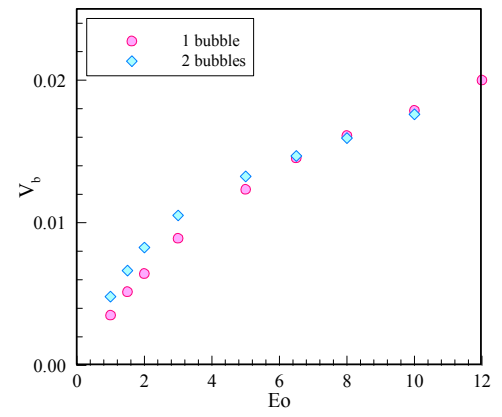

FIGURE IV. THE TERMINAL RISING VELOCITY FOR DIFFERENT EOTVOS NUMBERS.

\section{CONCLUSION}

In this work the lattice Boltzmann method is used to simulate the motion of two bubbles under gravity. The Laplace lawhas beenadopted to validate the present method.Thevertical arrangementis taken into account in the simulations withEotvos number ranging from 1 to 12 . The uppermost bubble deforms the most because of the maximum drag. In addition, after the coalescence a larger bubble is formed eventually. The terminal velocity of two bubbles is a little larger than that of one bubble under the same conditions.However, the terminal velocity is similar for all cases when the Eotvos number is large.

\section{ACKNOWLEDGEMENTS}

This work was supported by the National Program on Key Basic Research Project of China (grant no. 2011CB706501) and the National Natural Science Foundation of China (grant no. 11272302).

\section{REFERENCES}

[1] Dijkink, R. J., van der Dennen, J. P., Ohl. C. D., Prosperetti, A., The 'acoustic scallop': a bubble-powered actuator.Journal of Micromechanics and Microengineering, 16, pp. 1653-1659, 2006.

[2] Van Wijngaarden, L., Vossers, G., Mechanics and physics of gas bubbles in liqiuids: a report on Euromech 98.Journal of Fluid Mechanics, 87, pp. 695-704, 1978.

[3] Batchelor, G.K., The stability of a large gas bubble rising through liquid.Journal of Fluid Mechanics, 184, pp. 399-422, 1987.

[4] Krishna, R., van Baten, J. M., Simulating the motion of gas bubbles in a liquid.Nature,398, pp. 208, 1999.

[5] Joseph, D.D., Rise velocity of a spherical cap bubble.Journal of Fluid Mechanics, 488, pp. 213-223, 2003.

[6] Takada, N., Misawa, M., Tomiyama, A., Hosokawa, S., Simulation of bubble motion under gravity by Lattice Boltzmann Method.Journal of Nuclear Science and Technology, 38(5), pp.330-341, 2001.

[7] Shan, X., Chen, H., Lattice Boltzmann model for simulating flows with multiplephases and components.Physical Review E, 47, pp. 1815-1819, 1993.

[8] Rothman, D.H., Keller, J.M., Immiscible cellular-automaton fluids.Journal of Statistical Physics, 52, pp. 1119-1127, 1988.

[9] Swift, M.R., Osborn, W.R., Yeomans, J.M., Lattice Boltzmann simulation of non-ideal fluids.Physical Review Letter, 75, pp. 830-833, 1995.

[10] Zheng, H.W., Shu, C., Chew, Y.T.,A lattice Boltzmann model for multiphase flows withlarge density ratio.Journal of Computational Physics, 218, pp. 353-371, 2006.

[11] Gupta, A., Kumar, R.,Lattice Boltzmann simulation to study multiple bubble dynamics. InternationalJournal of Heat and Mass Transfer, 51, pp. 5192-5203, 2008.

[12] Yu, Z., Yang, H., Fan, L.S.,Numerical simulation of bubble interactions using an adaptive lattice Boltzmann method.Chemical Engineering Science, 66(14), pp. 3441-3451, 2011. 